\title{
Photorefractive Polymer System with a Low Glass Transition Temperature for a Holographic Recording
}

\author{
Nam-Jun Kim, Hyunaee Chun, In Kyu Moon, Won-Jae Joo, and Nakjoong Kim \\ Center for Organic Photorefractive Materials, Deparment of Chemistry, Hanvang Lniversity, Seoul 133-791, Korea \\ Received December 20,2001
}

\begin{abstract}
The photorefractive polymeric composite with good performance was prepared. The carbazole-substituted polysiloxane sensitized by 2.4.7-trinitro-9-fluorenone was used as a photoconducting medium and 1-[4-(2nitroviny l)pheny l]piperidine was added as an optically nonlinear chromophore. The photorefractive property of polymer was determined by diffraction efficiency using a $100 \mu \mathrm{m}$-thick film. The maximum diffraction efficiency $\left(\eta_{\text {max }}\right)$ of $71 \%$ was obtained at the electric field of $70 \mathrm{~V} / \mu \mathrm{m}$. The potential of the current polymer material as a holographic recording medium was evaluated by the demonstration of holographic recording and subsequent reading of optical image.
\end{abstract}

Keywords : Photorefractive polymer, Diffraction efficiency. Holography, Application.

\section{Introduction}

In the $21^{\text {st }}$ century of the information age. demands for the advanced information technology are drastically increasing. Thus the contribution of optical teclunology' to the advanced computer networks and telecommunication sy stems becomes more and more important. With the advance of the optical teclunology. the research on development of the new photonic material. which possesses high sensitivity. high efficiency. high optical clarity. low cost and high processability. has been stimulated. Photorefractive polymer has been recognized as one of the promising photonic materials to meet these requirements.

Photorefractivity is based on the combined effects of photoconductivity and electrooptic property. ${ }^{23}$ Under the illumination of non-uniform light formed by the interference of two coherent laser beams. a spatially oscillating spacecharge field is formed arising from the generation and the redistribution of photo-induced charges. The refractive index of material is subsequently modulated via an electrooptic effect. The photorefractive materials exhibit the unique features such as photosensitivity. reversibility and beam amplification. which provide the important advantages for their applications in the fields of optical data storage and information processing. ${ }^{1-5}$

In 1991 the polymeric photorefractive material was first discovered in nonlinear optical polymer. bisphenol A diglycidyl diether 4-nitro-1,2-phenylenediamine (bisA-NPDA). doped with the hole transporting agent. (diethylamino)benzaldehyde diphenylhydrazone. "The first polymeric photorefractive material exhibited the diffraction efficiency $(\eta)$ of $5 \times 10^{-5} \%$ and the gain coefficient $(\Gamma)$ of $0.33 \mathrm{~cm}^{-1}$. However. the performance of polymer materials has been significantly improved within the short research period. ${ }^{7-10}$ The poly- $N$-vinylcarbazole (PVK) composite doped with 2.5 dimethyl-(4-p-nitrophenylazo)anisole (DMNPAA. $50 \mathrm{wt} \%$ )

\footnotetext{
Conesponding Author. E-mail: kinnjatahanyang. ac.kr
}

chromophore. ethylcarbazole (ECZ, $16 \mathrm{wt} \%$ ) as a plasticizer. and 2.4.7-trinitro-9-fluorenone (TNF, $1 \mathrm{wt} \%$ ) as a charge sensitizer showed the large gain coefficient of $220 \mathrm{~cm}^{-1}$ and the diffraction efficiency of nearly $100 \%$ at the applied electric field of $90 \mathrm{~V} / \mu \mathrm{m} .{ }^{7}$ More recently. the several photorefractive polymers. which possess better properties than DMNPAA composite have been reported ${ }^{89.16}$ Despite of these progresses. however. the search for new photorefractive materials is still required for their widespread utilization in information teclnologies.

Here we report the photorefractive polymer system possessing a large modulation of refractive index (i.e. high photorefractive property) and high device stability using a simple preparation method. For this purpose. the new chromophore with high electrooptic activity and compatibility with matrix polymer were synthesized and the photorefractive guest-host system were prepared. Photorefractive property and the potential as a holographic recording medium of the current polymer șistem were investigated.

\section{Experimental Section}

Materials. The chemical structures of molecules used in this work are shown in Figure 1. The photorefractive material with a low glass transition temperature ( $T_{g}$ ) was prepared by doping 1-[4-(2-1itroviny 1$)$ pheny l]piperidine (P-ST-NO) cluromophore into photoconducting polymer matrix poly [methyl-3(9-carbazolyl) propylsiloxane] (PSX-CZ) sensitized by 2,4.7-trinitro-9-fluorenone (TNF). PSX-CZ was synthesized by the previously described method ${ }^{11}$ and TNF (Kanto Chem. Co. Inc.) was used after purification. P-ST-NO chromophore was synthesized as described in the following paragraph. Chemical structure of the molecule was characterized by an NMR spectrometry (Varian. Mercury $400 \mathrm{MHz}$ ). The absorption maximum $\left(\lambda_{\max }\right)$ of chromophore was determined by an UV-VIS spectrophotometry (Optizen 2120). The thermal transition temperatures of chromophore and composite were determined by differential scauning calorimetry (Perkin- 


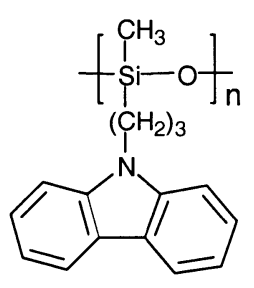

PSX-CZ<smiles>O=[N+]([O-])C=Cc1ccc(N2CCCCC2)cc1</smiles>

P-ST-NO

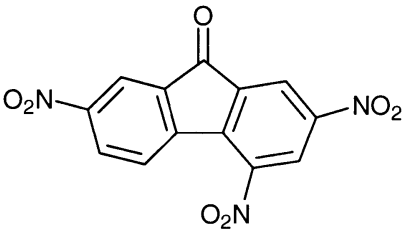

TNF
Figure 1. Chemical structure of components of photorefractive composite. Poly [methy 1-3-(9-carbayolyl) propylsiloxanel (PSX-C\%). 2.4.7-trinitro-9-tluorenone (TNF), and $1-\mid 4-(2-$-nitroviny l) phenyl|pipcridinc (P-ST-NO).

T:Imer, DSC7) at the heating rate of $10^{\circ} \mathrm{C} / \mathrm{min}$.

Synthesis of 1-|4-(2-Nitrovinyl)phenyl|piperidine (PST-NO). Piperidino-benzaldehyde was prepared from the reaction of 4-fluorobenzaldehyde and piperidine in dimethylformamide, as described elsewhere. ${ }^{12}$ A solution of 4 piperidinobenzaldehyde $(5 \mathrm{~g}, 0.026 \mathrm{~mol})$ and nitromethane $(2.1 \mathrm{~g}, 0.034 \mathrm{~mol})$ in acetic anhydride $(10 \mathrm{~mL})$ was refluxed for 6 hours under nitrogen and then the resulting solution was cooled to room temperature. After the solvent removal, the residue was purified by recrystallization from the mixed solvent of THГ : hexane $(=1: 3$ by volume ratio). The reaction product was obtained as a bright-red solid after purification. Yield: $82 \%$. Mp: 112 "C. $\lambda_{\text {max }}: 438 \mathrm{~nm}$. ${ }^{\prime} \mathrm{H}-$ NMR (CDCl, ppm) $\delta_{1}=1.68\left(\mathrm{br}, 6 \mathrm{H}_{,}, 3 \times \mathrm{CH}_{2}\right), 3.38(\mathrm{t}, 4 \mathrm{H}$, $\left.2 \times \mathrm{CH}_{2}\right), 6.82\left(\mathrm{~d}, 2 \mathrm{H}, \mathrm{H}_{\text {plienyly }}\right), 7.41\left(\mathrm{~d}, 2 \mathrm{H}, \mathrm{H}_{\text {pherryll }}\right), 7.50(\mathrm{~d}$, IH, $\left.\mathrm{H}_{\text {siny }}\right), 7.92$ (d, $\left.1 \mathrm{H}, \mathrm{H}_{\mathrm{s} \text { iny }}\right)$ ).

Sample Preparation. The composition of photorefractive material was PSX-C7. : P-ST-NO : TNF $=69: 30: 1$ by wt\%. As shown in Figure 2, the glass transition temperature $\left(T_{y}\right)$ of this composite determined by differential scaming calorimetry was $27^{\circ} \mathrm{C}$, which assures the facile orientation of dipolar chromophore at the measuring temperature of $28^{\circ} \mathrm{C}$. Note that in the case of low $\mathrm{J}_{\mathrm{g}}$ material, the glass transition temperature should be in the vicinity of or lower than measuring temperature in order to take advantage of orientational enhancement effect. ${ }^{5-10.13}$

The predetermined amounts of components were dissolved in dichloromethane and the resulting solution was filtered through a $0.2 \mu \mathrm{m}$ filter. The composite was casted on indium tin oxide ( $1 \mathrm{TO}$ ) glass plate, dried for 6 hours at ambient temperature and subsequently heated in an oven at $90^{\circ} \mathrm{C}$ for 24 hours to remove the residual solvent completely. 'Then, composite was softened by placing it on a hot plate at $100^{\circ} \mathrm{C}$ and then covered with the second 110 coated glass. The thickness of film was controlled by 'Jeflon spacer between two ITO glass plates.

Measurements. The electrooptic property of the polymeric composite was determined by transmission ellipsometric method. ${ }^{14}$ The sample tilted by $45^{\circ}$ was placed between the polarizer and the analyser with the polarization set to $-45^{\circ}$ $-45^{\circ}$, respectively. The electric-field induced birefringence $(\Delta \mathrm{n})$ of composite was determined from the variation of the

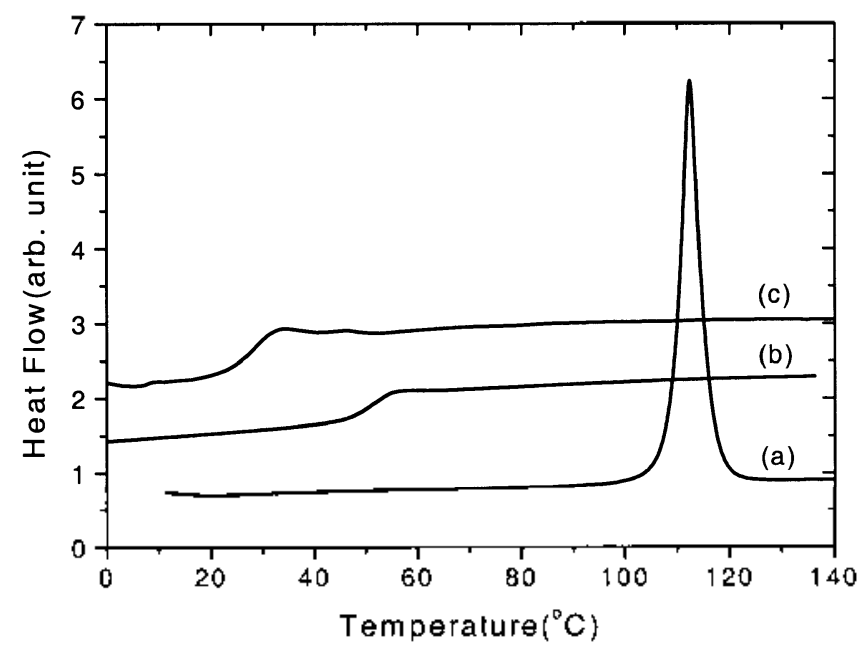

Figure 2. Thermal transition temperatures determined by differential scanning calorimetry: (a) Melting temperature $\left(T_{10}\right)$ of $P-S T$ NO chromophore. (b) Glass transition temperalure ( $T_{n}$ ) of PSXC\%.. (c) Glass transition lemperature ( $\mathrm{I}_{2}$ ) of photorel ractive composite. The composition is $\mathrm{PSX}-\mathrm{CZ}: \mathrm{P}-\mathrm{ST}-\mathrm{NO}: \mathrm{TNF}=69$ : $30: 1$ by $* 4 \%$. The heating rate is $10^{\circ} \mathrm{C} / \mathrm{min}$.

transmitted intensity ( $T$ ) through crossed polarizers upon the application of electric field, as described by the following equation (1).

$$
T=\sin ^{2}\left(\frac{2 \pi}{\lambda} \cdot l \cdot \Delta n\right)
$$

where $\lambda$ is wavelength and $l$ is a distance of light path.

The diffiaction efficiency of photorefractive material was determined by the degenerate four wave mixing (DFWM) experiment. Two coherent laser beams at the wavelength of $632.8 \mathrm{~nm}$ were irradiated on the sample in the tilted geometry with the incident angle of $30^{\circ}$ and $60^{\circ}$ with respect to sample normal. The writing beams both were $s$-polarized and had the equal intensity of $60 \mathrm{~mW} / \mathrm{cm}^{2}$. The recorded photorefractive grating was read out by a $p$-polarized counter-propagating beam. Attenuated reading beam with the very weak intensity of $0.1 \mathrm{~mW} / \mathrm{cm}^{2}$ was used. The internal diffraction efficiency $\left(\eta_{\text {int }}\right)$ of photorefractive material was determined from equation $(2)^{7}$

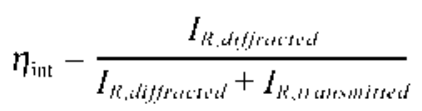

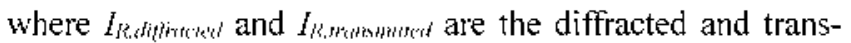
mitted intensities of reading beam, respectively.

Holographic application of polymeric material was performed at the wavelength of $632.8 \mathrm{~nm}$ using the setup shown in Figure 3 . The s-polarized laser light was split into object and reference beams. The spatially filtered object beam was expanded into $30 \mathrm{~mm}$ in beam diameter and then was passed through the mask with a dimension of $15 \mathrm{~mm} \times 15 \mathrm{~mm}$. The hologram was recorded in the photorefractive sample by intersecting of object beam containing the information and reference beam. The incident angles of the object and refer- 


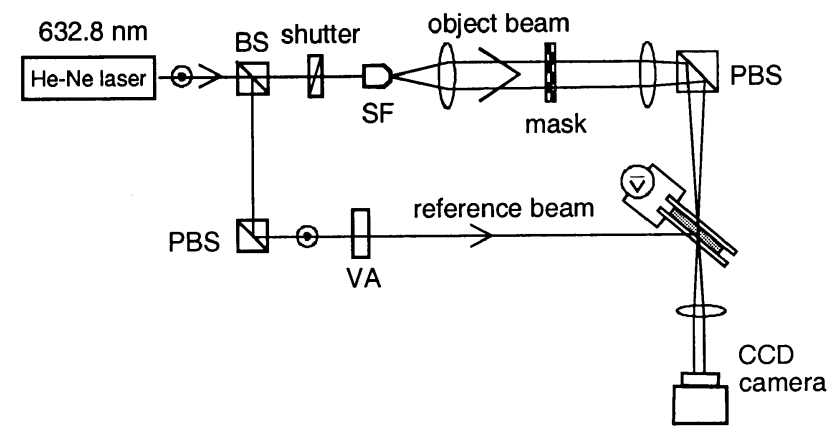

Figure 3. Schematic diagram of optical setup used for holographic applications: BS: nonpolarizing heam splitter. PBS: polarizing beam splitter. SF: spatial lilter. VA: variable attenuator. C(CD: charge-coupled-device camera.

ence beams were $45^{\circ}$ and $135^{\circ}$ with respect to sample normal, respectively. The internal angle of $90^{\circ}$ was used in order to maximize the angular selectivity. ${ }^{15}$ The recorded hologram inage was read back by illuminating of $s$-polarized attenuated light with the intensity of $0.01 \mathrm{~mW} / \mathrm{cm}^{2}$ and the reconstructed hologram image was recorded on a chargecoupled device (CCD) camera.

\section{Results and Discussion}

Design of Optically Nonlinear Chromophore. Since the refractive index modulation and the device stability of photorefractive material are largely governed by the chromophore embedded in photoconducting medium, the preparation of the optically nonlinear chromophore is of critical importance. ${ }^{\text {. } 10.16}$ In this work, we synthesized 1-[4-(2-nitrovinyl)phenyl]piperidine (P-ST-NO) as a chromophore for photorefractive application. The design strategy of this chromophore is to prepare the molecule which gives rise to the large orientational birefringence, since the contribution of orientational birefringence on the steady-state photorefractive property of the low $\mathrm{J}_{2}$ material is much greater than that of the l'ockels effect. ${ }^{16.17,18}$ Based on the semiempirical quantum chemical calculation method. MOPAC 6 with the PM3 procedure for geometry optimization, the dipole moment and the polarizability anisotropy of $\mathrm{P}-\mathrm{S} \mathrm{I}-\mathrm{NO}$ chromophore are 8.0 Debye and 153 A.U., respectively. Thus it is expected that the large dipole moment and the moderate length of P'ST-NO chromophore may facilitate the molecular alignment along the electric field and the relatively large polarizability anisotropy will be beneficial to the orientational birefringence of photorefractive material. ${ }^{18}$

Electrooptic and Photorefractive Properties. Field-induced birefringence of the photorefractive polymer system was characterized by transinission ellipsometric measurement at various applied fields. As given in Figure 4, birefringence $(\Delta \mathrm{n})$ of composite containing $30 \mathrm{wt} \%$ of $\mathrm{P}-\mathrm{S} \mathrm{C}-\mathrm{NO}$ chromophore is quadratically increased with the applied fields. At $70 \mathrm{~V} / \mu \mathrm{m}$, the value of $\Delta \mathrm{n}$ is $c a .2 .0 \times 10^{-3}$, which is the sufficient large value for the preparation of the efficient photorefractive materials. ${ }^{14.19} \mathrm{Be}$ reminded that this mea-

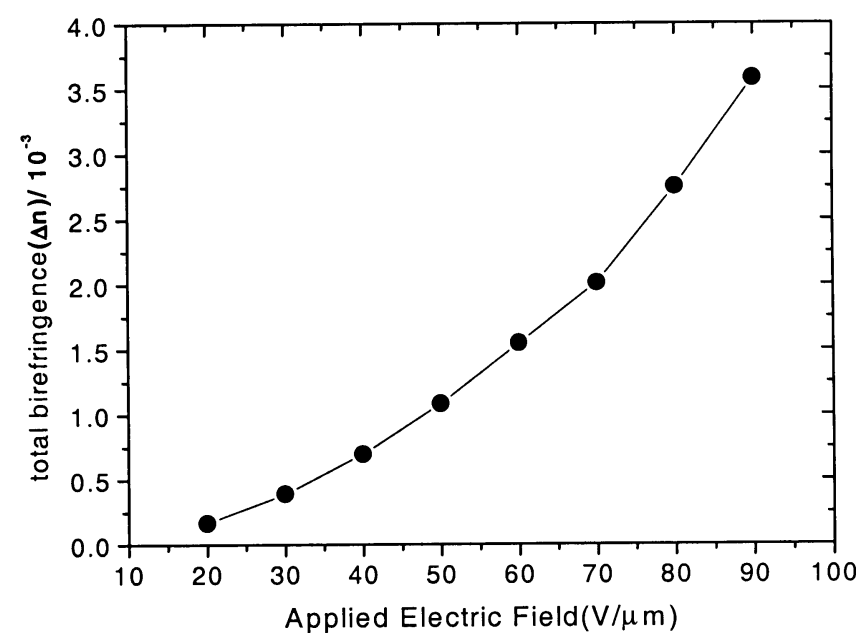

Figure 4. Llectric tield induced birefringence of P-NO-ST composite at various applied clcetric fields.

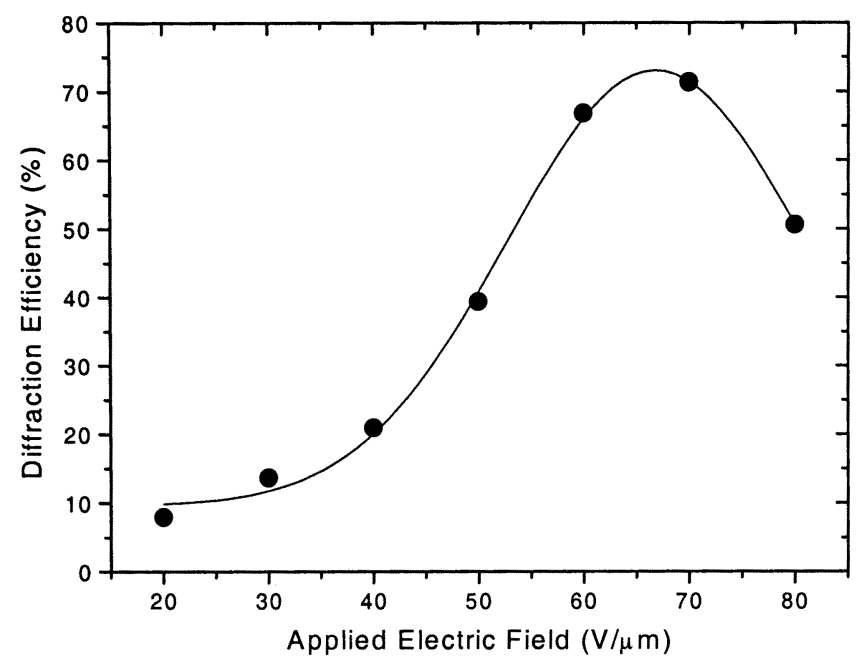

Figure 5. Steady-state diffraction efficiency of P-NO-ST composite at various applied electric fields.

surement provides the useful information in the evaluation of chromophore, since the field-induced Kerr nonlinearity was determined without complication by the space-charge field. ${ }^{14}$

The performance of photorefractive material was evaluated by diffraction efficiency measured by DFWM method. The variation of diffraction efficiency with a function of an applied electric field was determined using a $100 \mu \mathrm{m}$-thick film. As shown in Figure 5, the current P-ST-DC composite shows the maximum diffraction efficiency ( $\eta_{\max }$ ) of $71 \%$ at $70 \mathrm{~V} / \mathrm{mm}$. The diffraction efficiency $(\eta)$ of composite shows an oscillatory behavior, reflecting the sin square dependency: $:^{3.2 !}$

$$
\eta=\sin ^{2}[C \cdot \Delta n]
$$

where $\Delta n$ is the refractive index modulation and $C$ is the constant determined by a wavelength, thickness, geometry of sample and so on. 


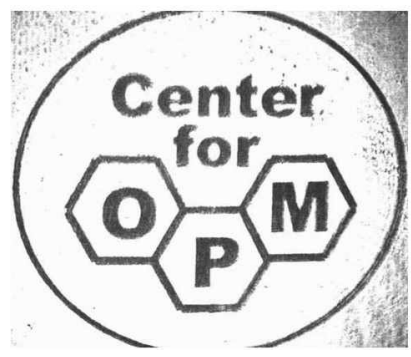

(a)

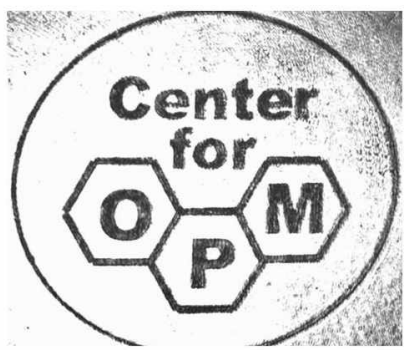

(b)
Figure 6. Photographs of (a) original image transmitted through the sample and $(b)$ reconstructed hologram image

Application of Photorefractive Polymer to Holography. The potential of our composite as a holographic recording medium was assessed using $100 \mu \mathrm{m}$ thick composite. Figure $6(a)$ is the original inage transmitted through the sample. Hologram image was recorded at the external field of $60 \mathrm{~V} /$ $\mu \mathrm{m}$ and the stored optical information is reconstructed in real-time operation by illuminating of probing beam. As shown in Figure 6(b). the reconstructed hologram image has the good contrast. being comparable to original image. The recorded hologram can be completely erased by illunination of reference beam alone. This experimental result proves the performance of the current polymeric material as an efficient holographic recording medium.

Device Stability. Among the many polymeric photorefractive materials reported so far. guest-host sy stems based on a photoconducting polymers doped with optically nonlinear chromophores have been extensively studied due to their promising properties. However, guest-host systems often suffer from the phase separation associated with the limited compatibility of polar chromophore with the photoconducting polymer and the high concentration of dopants..$^{3 i}$

Our polymer sample containing $30 \mathrm{wt} \%$ of P-ST-NO chromophore shows the good phase stability. The optical clarity of device has been retained over several months when stored at roon temperature, which was confirmed by an UVVIS spectrometer. This may arise partially from the good compatibility of P-ST-NO chromophore with matrix polymer. which can be assured from the observation that the melting peak of P-ST-NO chromophore disappears completely in photorefractive polymer composite (see Figure 2). Furthermore. the lower concentration of dopants in our material may also contribute to the device stability. being compared with typical photorefractive gnest-host system. ${ }^{7 \times 9.20}$ No extra plasticizer was added in our composite, due to the relatively low $\mathrm{T}_{\mathrm{g}}\left(5 \mathrm{I}^{\circ} \mathrm{C}\right)$ of $\mathrm{PSX}-\mathrm{CZ}$. $\mathrm{T}_{\mathrm{g}}$ of our photorefractive polymer can be lowered to room temperature sumply by adding the chromophore. differently from PVK composite which generally contain plasticizer in the range of 15 to $20 \mathrm{wt} \%$.

\section{Conclusions}

In this work we prepared the new photorefractive with low glass transition temperature possessing a large modulation of refractive index and high device stability. The photorefractive composite containing the $30 \mathrm{wt} \%$ of P-ST-NO showed the high diffraction efficiency of $71 \%$ at the electric field of $70 \mathrm{~V} / \mathrm{um}$. The holographic application of photorefractive polymer was successfully demonstrated. which proves the potential of our material as an efficient holographic recording medium in the field of optical information processing.

Acknowledgment. This work was financially supported by Hanyang University, Korea made in the program year of 2000 .

\section{References}

1. Solymar. L:: Webb. D. T.: Grunnet-Tepsen. A. In The Phusics and Applications of Photoreffactive Material, Oxtord, U. K, 1996.

2. Gunter. P. Huignard. J.-P. In Photorafractive Hatarals and Their Applications I and II, Springer-Verlag: Berlin. Germany, 1988 (vol.I). 1989 (vol.II).

3. Moerner. W. E.: Silence. S. M. Chent. Rev: 1994. 94. 127.

4. Agullo-Lopez. F.: Cabrera. I. M.: Agullo-Rueda. F. In Electrooptics: Academic Press: London. 1994: chap 10, chap 11.

5. Lundquist, P. M. Wortmann. R: Geletnehy, C. Tweig, R. J.: Jurich. M.: Lee. V. Y.: Moylan. C. R.: Burland. D. M. Science? 1996. 274. 1182 .

6. Ducharme. S.: Scott. J. C.: Twieg. R. J.: Moerner W. E. Plns. Rev: Lett. 1991. 66. 1846.

7. Meerholtz, K.: Volodın, B. L: Sandalphon: Kippelen, B.: Peyghambarian. N. Nature 1994, 371,497.

8. Wright. M.: Diaz-Gareia. M. A.: Casperson. J. D.: DeClue, M.: Moerner. W. E. Appl. Phws. Leth 1998. 73 . 1490.

9. Wurthner. F.: Yao. S.: Schilling. T.: Wortmann. R.: Redi-Abshiro. M.: Mecher. E.: Gallego-Gomez. F.: Meerholz. K. J. An Chen. Soc. $2001,123,2810$

10. Chun. H.: Moon, I. K.; Shın, D. H.: Kim. N. Chem. Mater. 2001. 13. 2813 .

11. Strohriegl. P. Machrontol Chen. Rapid Conmum. 1986. 7.771

12. Silence. S. M.: Donckers. M. C. J. M.: Walsh. C. A.: Burland. D M.: Twieg. R. T. Moerner. W. E. Appl Opt 1994. 33. 2218.

13. Moerner, W. E.: Silence, S. M: Hache, F; Bjorklund. G. C. J. Opt. Soc. Am. B 1994. 11, 320.

14. Bittner. R.: Daubler. T. K; Neher, D; Meerholz, K. Adv Mater. 1999. H. 123.

15. Hong. J. H.: McMichael. I.: Chang. T. Y.: Christian. W.: Paek. E. G. Opt. Eng. 1995. 3+. 2193.

16. Wortmann, R.; Poga. C.: Twieg, R. J:- Geletneky. C. C.: Moylan, R.; Lundqust, P. M: DeVoe. R. G; Cotts. P. M: Horn, H.: Rice. J. E.) Burland, D. M. J. Chem. Phys. 1996. 105. 10637.

17. Kippelen. B.: Meyers. F.: Peyghambarian. N.: Marder. S. R. J. An Chen Soc. 1997. 119. 4559.

18. Moylan1. C.: Wortmant1. R.: Twieg. R. T.: McComb. I. H. J. Opt. Soc. Am. B 1998. 15,929.

19. Chun. H.: Moon. I. K; Kım, N.: Shin, D.-H.: Song, S. J. Mater: Chem 2001, submitted

20. Zilker. S. J. Chemplnchent 2000. 1. 72. 